\title{
Reprodutibilidade de Experimentos sob Irradiação de Micro-ondas
}

Marcella C. Flores, Carla C. Diederichs, Louise F Matos, Leandro S. M. Miranda \& Rodrigo O. M. A. Souza

A utilização de irradiação de micro-ondas tem se difundido entre os químicos orgânicos sintéticos. No entanto, o cuidado quanto à reprodutibilidade e ao correto uso dos equipamentos pode vir a ser no futuro um entrave à utilização dos mesmos. Sendo assim, neste trabalho viemos esclarecer alguns pontos e demonstrar como alguns cuidados podem fazer diferença durante o processo de irradiação de micro-ondas.

Palavras-chave: irradiação de micro-ondas, efeito térmico, efeito de micro-ondas, temperatura.

The use of microwave irradiation has been widespread among synthetic organic chemists. However, proper care about the reproducibility and the correct use of equipment may become an obstacle to the future use of this technology. Thus, in this work we would like to clarify some points and show how the adequate use of temperature measurement can make a difference during the microwave irradiation.

Keywords: microwave irradiation, thermal effect, microwave effect, temperature. 


\section{Introdução}

A irradiação de micro-ondas aplicada à Química Orgânica vem ganhando, ao longo dos últimos anos, cada vez mais espaço entre os químicos orgânicos sintéticos, fato este que pode ser constatado devido ao grande número de publicações relacionadas ao tema que podem ser encontradas em revistas de alto impacto como Journal of Organic Chemistry, Organic Letters, Green Chemistry, entre outras, e também pela inserção deste tipo de técnica dentro do escopo das disciplinas de Química Orgânica Experimental em diversas universidades brasileiras.

Desde os primeiros experimentos feitos por Gedye e Guiguere ${ }^{1}$, foi possível observar que a tecnologia de irradiação de micro-ondas promovia um aumento da velocidade de reação e redução de formação de produtos colaterais, quando comparada com experimentos sob aquecimento convencional, fato este provavelmente relacionado ao rápido aquecimento e resfriamento do meio reacional.

A grande vantagem da utilização da irradiação de microondas em síntese orgânica é a capacidade de se promover um rápido aquecimento do meio reacional. Este aquecimento promovido pela irradiação de micro-ondas pode ser obtido basicamente por dois mecanismos: polarização dipolar e condução iônica. Desta maneira, quando uma reação é irradiada com micro-ondas, os dipolos ou íons presentes na mistura reacional alinham-se ao campo elétrico aplicado. Como o campo elétrico oscila, os dipolos ou íons tendem a se realinhar ao campo elétrico oscilante e neste processo perdem energia sob a forma de calor, devido aos choques moleculares e perdas dielétricas. Sendo assim, este tipo de aquecimento está intimamente relacionado às propriedades dielétricas dos solventes e reagentes. $\mathrm{O}$ aquecimento do meio reacional é dito volumétrico, pois acontece do interior da mistura reacional na direção das paredes do vaso, com manutenção da temperatura pré-determinada pelo operador quando da utilização de sensores de temperaturas presentes em reatores dedicados.

As micro-ondas são formadas por ondas eletromagnéticas com frequência entre 0,3 e $300 \mathrm{GHz}$, correspondendo a comprimentos de onda da ordem de $1 \mathrm{~cm}$ a 1 m e encontram-se na região do espectro eletromagnético entre o infravermelho e as radiofreqüências².

No que diz respeito à energia associada a esta onda eletromagnética, podemos observar na Tabela 1 que as micro-ondas possuem uma energia muito baixa, diferentemente do observado para a radiação ultravioleta, utilizada nos processos fotoquímicos ${ }^{3}$.

Tabela 1. Energia associada às micro-ondas e a algumas ligações químicas ${ }^{3}$

\begin{tabular}{|c|c|c|c|c|}
\hline Radiação & $\begin{array}{c}\text { Frequência } \\
\text { (GHz) }\end{array}$ & $\begin{array}{c}\text { Energia } \\
(\mathrm{eV})\end{array}$ & $\begin{array}{l}\text { Ligação } \\
\text { Química }\end{array}$ & $\begin{array}{c}\text { Energia } \\
(\mathrm{eV})\end{array}$ \\
\hline Raios Gama & 3,0 x 1011 & $1,24 \times 106$ & C-C & 3,61 \\
\hline Raios X & $3,0 \times 1010$ & $1,24 \times 105$ & $\mathrm{C}=\mathrm{C}$ & 6,35 \\
\hline Ultravioleta & $1,0 \times 106$ & 4,1 & $\mathrm{C}-\mathrm{O}$ & 3,74 \\
\hline Luz Visível & $6,0 \times 105$ & 2,5 & $\mathrm{C}=\mathrm{O}$ & 7,71 \\
\hline Infravermelho & $3,0 \times 103$ & 0,012 & $\mathrm{C}-\mathrm{H}$ & 4,28 \\
\hline Micro-ondas & 2,45 & 0,0016 & $\mathrm{O}-\mathrm{H}$ & 4,80 \\
\hline Radiofrequência & $1,0 \times 10-3$ & $4,0 \times 10-9$ & Lig. de $\mathrm{H}$ & 0,04 \\
\hline
\end{tabular}

O fato da utilização da irradiação de micro-ondas em reações orgânicas ter levado uma série de reações a terem seus tempos reacionais reduzidos de dias para minutos ou horas fez com que diversos grupos trabalhassem em busca de respostas para este efeito benéfico da utilização de micro-ondas, estando estes racionalizados através de três diferentes propostas, enumeradas a seguir:

- Efeitos Térmicos

- Efeitos Específicos de Micro-ondas

\section{- Efeitos Não-Térmicos de Micro-ondas}

Os efeitos térmicos podem ser entendidos como conseqüência direta das altas temperaturas reacionais que podem ser obtidas quando reagentes e solventes polares são irradiados com micro-ondas. A diminuição dos tempos de reação em temperaturas elevadas pode ser prevista pela aplicação da Lei de Arrhenius [ $\left.k=\mathrm{A} \exp \left(-E_{a} / \mathrm{R} T\right)\right]^{4}$.

Os efeitos específicos de micro-ondas podem ser conceituados como todos aqueles efeitos térmicos que não podem ser reproduzidos pelo aquecimento convencional. Neste contexto, encontram-se o super aquecimento de solventes à pressão atmosférica, aquecimento seletivo de reagentes e formação de "hot spots" (pontos isolados de alta temperatura no meio reacional). É importante ressaltar que apesar destes efeitos serem produzidos exclusivamente na presença da irradiação de microondas, seus resultados observados são função do aumento da temperatura reacional ${ }^{5}$. 
Os efeitos não-térmicos de micro-ondas correspondem a todos aqueles efeitos que não podem ser explicados como térmicos ou específicos de micro-ondas. A racionalização destes efeitos não-térmicos é feita basicamente através de duas abordagens: pelo aumento do fator pré-exponencial $A$ da equação de Arrhenius e pela diminuição do $\Delta \mathrm{G}$ de ativação do estado de transição. O primeiro baseia-se no aumento da probabilidade de choques intermoleculares devido às sucessivas mudanças de orientação das moléculas polares devido à oscilação do campo elétrico. O segundo sugere que mecanismos polares com formação de espécies carregadas no estado de transição deverão ser favorecidos pela irradiação de micro-ondas devido à interação com o campo eletromagnético gerado ${ }^{6}$.

No entanto, em ambos os casos (irradiação de microondas e aquecimento convencional) o argumento a ser utilizado para descrever determinado efeito deverá sempre estar bem embasado por dados referentes à temperatura do meio reacional, o que conduz diretamente à questão da aferição da temperatura do meio reacional.

Em geral, durante um experimento realizado sob aquecimento convencional, a aferição e controle da temperatura normalmente são feitos no banho de aquecimento (óleo, água, manta, etc) através de um Thermo-Par ou Termômetro de Mercúrio, porém, como é de conhecimento geral, esta temperatura não reflete o panorama da temperatura no meio reacional que facilmente pode chegar a $20^{\circ} \mathrm{C}$ de diferença, dependendo do volume e dos componentes do meio reacional.

Aqueles que acham que sob a irradiação de microondas em equipamentos dedicados este problema está contornado, muito cuidado. Os equipamentos hoje disponíveis no mercado oferecem diferentes configurações para aferição da temperatura reacional, como por exemplo, o sensor de infravermelho, a fibra ótica e o sensor de rubi. Cada um destes dispositivos possui uma característica específica e fornece informações de temperatura em tempo real ao usuário através da utilização de um software.

Uma vez que cada fabricante de reatores dedicados à irradiação de micro-ondas utiliza um sistema distinto para a mensuração da temperatura do meio reacional, as reações conduzidas sob esta tecnologia apresentam a questão da reprodutibilidade dos experimentos como tema central.

Neste contexto, o primeiro ponto a ser observado é a escolha do modo de monitoramento da temperatura. A maioria dos reatores dedicados mono modulares trabalha em um regime de controle da potência pelo feedback da temperatura reacional (temperature control mode), onde a potência do magnetron é regulada pela resposta oriunda do sensor de temperatura, permitindo que se chegue à temperatura selecionada com precisão. No entanto, dependendo do tipo de sistema utilizado para aferição da temperatura, respostas diferentes serão obtidas como poderemos verificar a seguir.

Naqueles reatores que operam com sensor de infravermelho, a temperatura é determinada através de um sensor externo, posicionado na parte inferior da cavidade e que afere a temperatura na parede do reator. No entanto, em geral, o frasco reacional não é transparente à radiação de micro-ondas, pois é feito em pyrex, levando a um aquecimento da superfície do vaso e, portanto não correspondendo à temperatura interna da reação, como podemos observar na Figura 1.

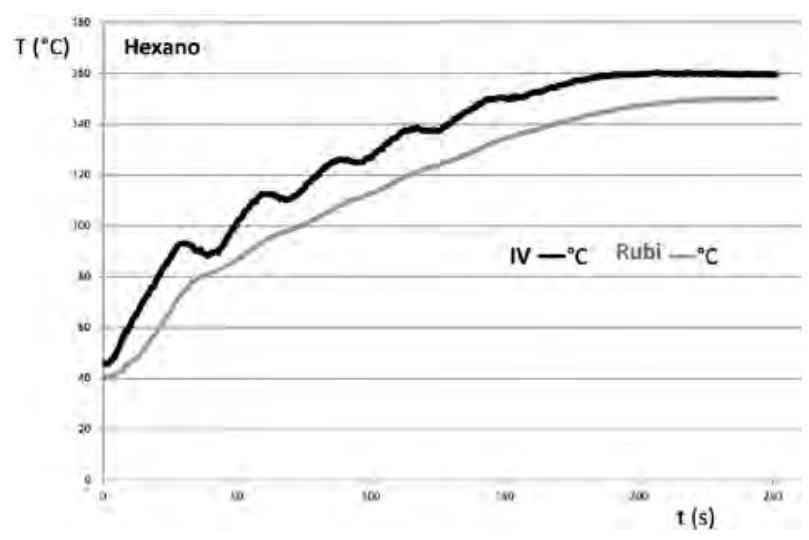

Figura 1. Aquecimento do hexano monitorado por sensor de infravermelho (IV) e de rubi.

Como pode ser visto na Figura 1, o aquecimento do hexano monitorado por diferentes sensores leva a temperaturas reacionais discrepantes durante todo curso reacional, podendo ser de até $20{ }^{\circ} \mathrm{C}$ em favor do sensor de infravermelho nos momentos iniciais do aquecimento. Isto acontece, pois o hexano, uma molécula de caráter apolar, tem um aquecimento mais lento quando comparado ao pyrex, levando assim a uma maior diferença entre as temperaturas internas (sensor de Rubi) e externas (sensor de IV) na fase inicial de aquecimento. Conforme a irradiação de micro-ondas se processa, o aquecimento do meio reacional também é feito pela transferência de calor das paredes do 
reator de pyrex para o solvente, chegando a uma menor diferença $\left(10^{\circ} \mathrm{C}\right)$ nos momentos finais de irradiação.

O panorama se torna um pouco diferente quando solventes mais polares, como por exemplo metanol, são utilizados. Devido à sua natureza polar, este solvente aquece rapidamente sobre influência da irradiação de micro-ondas, como pode ser observado na Figura 2. Neste caso, o sensor de infravermelho subestima a temperatura do meio reacional durante a fase inicial de aquecimento, podendo as diferenças de temperatura chegarem facilmente a $40{ }^{\circ} \mathrm{C}$, mostrando que para solventes desta natureza o aquecimento é mais rápido do que o aquecimento do pyrex.

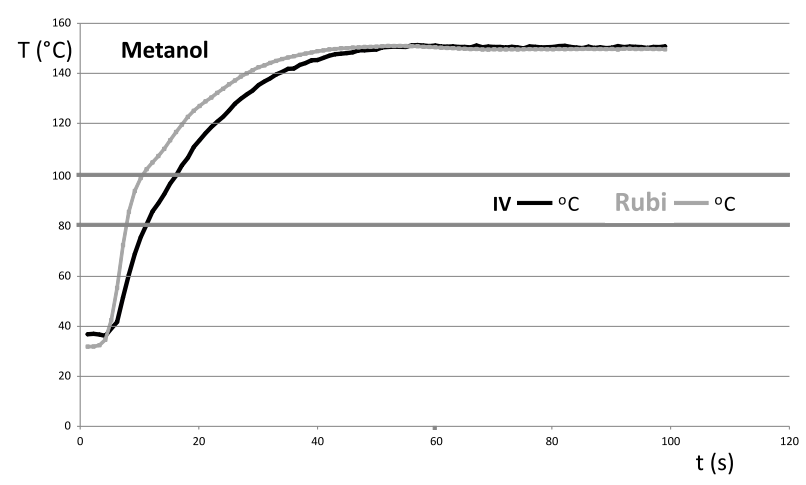

Figura 2. Aquecimento do metanol monitorado por sensor de infravermelho (IV) e de rubi.

No intuito de demonstrar estas diferenças, vários solventes foram submetidos ao aquecimento em equipamentos dedicados à irradiação de micro-ondas. $\mathrm{O}$ equipamento utilizado foi o Monowave 300, que permite o monitoramento em tempo real da temperatura do meio reacional através do sensor de infravermelho e rubi em paralelo. A rampa de aquecimento escolhida foi "as fast as possible”, na qual o equipamento realiza o aquecimento do meio reacional utilizando potência máxima.

Foram utilizados vasos reacionais de $10 \mathrm{~mL}$ com $3 \mathrm{~mL}$ de solvente; a temperatura escolhida foi de 150 ${ }^{\circ} \mathrm{C}$ e um período de aquecimento de 5 minutos. No Gráfico 1, está o tempo necessário (em segundos) para o aquecimento de diversos solventes até a temperatura estudada. Em preto, encontra-se o tempo necessário para atingir a temperatura estipulada medindo-se pelo sensor de rubi e, em cinza, os resultados obtidos pelo sensor de infravermelho. Entre parênteses encontram-se os valores de tangente de perda para cada um dos solventes.
Como esperado, solventes com alta tangente de perda $(\delta>0,5$; valor em parênteses no gráfico 1$)$ são aquecidos rapidamente pelas micro-ondas. Nestes casos, um aquecimento mais rápido é esperado através da mensuração da temperatura com sensor de Rubi quando comparado ao infravermelho. Vale a pena ressaltar que a tangente de perda é um parâmetro dielétrico, que quantifica a sua capacidade de dissipação de energia eletromagnética inerente. $\mathrm{O}$ termo refere-se à tangente do ângulo em um plano complexo entre a componente resistiva de um campo eletromagnético e suas componentes reativas.

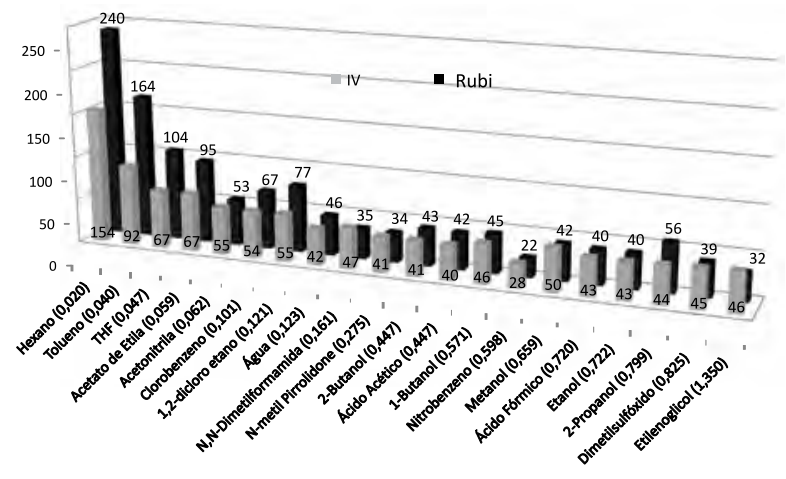

Grafico 1. Tempo necessário para diversos solventes atingirem a temperatura de $150{ }^{\circ} \mathrm{C}$ medido pelo sensor de rubi (preto) e infravermelho (cinza).

Chama a atenção também a diferença de tempo que os solventes com baixa tangente de perda possuem para chegar à temperatura do experimento, dependendo do modo como essa temperatura é mensurada. O hexano, segundo o Gráfico 1, levou 154 segundos para atingir a temperatura de $150{ }^{\circ} \mathrm{C}$, segundo monitoramento através de sensor de infravermelho. No entanto, o sensor de rubi, responsável por aferir a temperatura do meio reacional, ainda demorou 240 segundos para atingir a mesma temperatura, mostrando a diferença entre a temperatura obtida na parede do frasco e a temperatura do meio reacional, uma diferença de 86 segundos. Estes detalhes são de extrema importância em reações sob irradiação de micro-ondas, pois muitas reações podem levar apenas alguns segundos para se completar. Assim como no hexano, tolueno, acetato de etila e tetraidrofurano (THF) têm o interior do vaso aquecido por convecção a partir do aquecimento preferencial do vidro, o que conduz a uma falsa rampa de aquecimento do meio reacional através do sensor de infravermelho, visto que a transferência de calor do vidro para o meio reacional é mais 
lento que o aquecimento direto proporcionado pelas microondas da parede do vaso reacional. O mesmo não acontece quando frascos de quartzo são utilizados, pois estes são transparentes a irradiação de micro-ondas, fazendo com que a rampa de aquecimento seja mais lenta, pois dependerá basicamente do aquecimento dielétrico produzido pela interação da irradiação de micro-ondas com o solvente.

Solventes como etilenoglicol, dimetilsulfóxido, nitrobenzeno, metanol e etanol apresentaram este comportamento típico de solventes com alta tangente de perda. Entretanto, o caso do 2-propanol chama a atenção, pois este não apresenta o mesmo comportamento $(\delta=0,799)$.

No Gráfico 1, é possível observar que durante o período de aquecimento correspondente ao 2-propanol, a taxa de aquecimento medida pelo infravermelho assemelha-se ao comportamento de solventes com tangente de perda próximas como DMSO, etanol e ácido fórmico. Entretanto, segundo o sensor de rubi, este solvente apresenta um tempo superior para atingir a temperatura do experimento.

Quando as curvas de aquecimento medidas pelo IV e rubi para o isopropanol são comparadas, observa-se que naquela medida pelo sensor de rubi a taxa de aquecimento é menor quando comparada ao infravermelho (Gráfico 2).

Uma proposta para este comportamento do 2-propanol baseia-se na habilidade de determinada substância em absorver radiação de micro-ondas, a qual é decorrente de suas propriedades dielétricas e que estas são dependentes da temperatura.

Este decréscimo indica que à medida que o solvente é aquecido este se torna mais transparente à radiação de micro-ondas, o que decresce a sua taxa de aquecimento medida no interior do vaso. Infelizmente dados relativos à dependência das propriedades dielétricas em função da temperatura não existem para a maioria dos solventes. Entretanto, como no caso de solventes com alta tangente de perda o mecanismo de aquecimento se dá através da polarização dipolar onde os dipolos individuais tendem a se alinhar ao campo elétrico oscilante e o aquecimento ocorre através de fricção dipolar. Uma vez que o aumento da temperatura diminui a viscosidade do sistema, tornase possível um alinhamento livre de fricção, conduzindo a uma menor dissipação de calor e, em conseqüência, a uma menor taxa de aquecimento.

Além da potência e o modo como a temperatura é mensurada, a reprodutibilidade depende também de fatores intrínsecos aos equipamentos. Os sensores de rubi e fibra óptica, por estarem inseridos no meio reacional, apresentam uma resposta mais próxima da realidade no que diz respeito à medida da temperatura interna do vaso reacional, entretanto também possuem limitações, como já mencionado anteriormente. Estes sensores apresentam custo mais elevado sendo vendidos apenas como um acessório. Além disso, no intuito de aumentar a vida útil destes sensores, eles apresentam-se protegidos do meio reacional, o que pode propiciar um atraso na resposta, atraso este importante no caso de soluções que apresentem aquecimento muito rápido, como líquidos iônicos por exemplo.

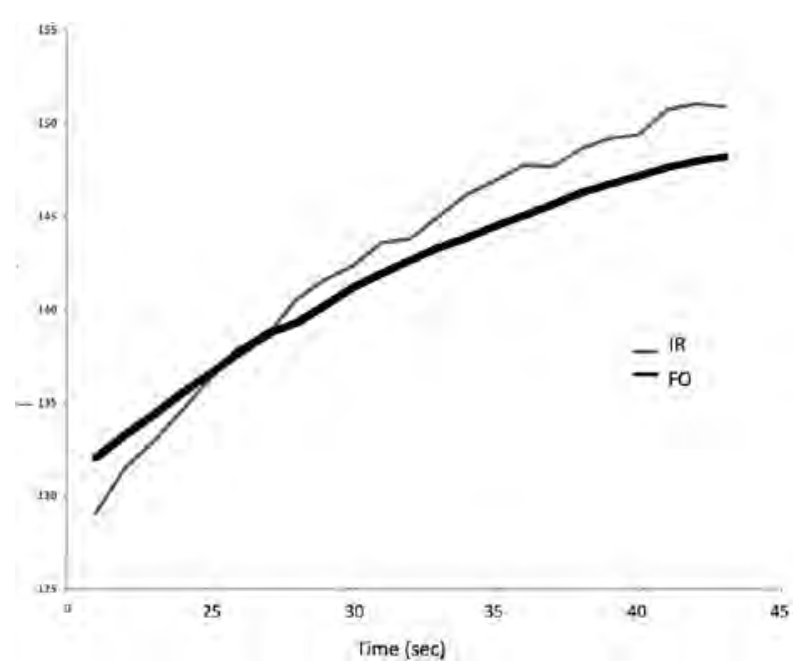

Gráfico 2. Curva de aquecimento do 2-propanol medido pelo sensor de infravermelho e de rubi.

Outra questão da qual é necessário tomar-se ciência quando o experimento não é reproduzido no mesmo reator é o fato de cada fabricante utilizar um algoritmo diferente para o processo de irradiação de micro-ondas. Isto faz com que a resposta do magnetron à informação de temperatura enviada pelo sensor seja diferente, especialmente quando não operando à potência constante, onde esta dificuldade é minimizada. Infelizmente, nem todos os equipamentos fornecem a possibilidade de operação à potência constante.

Com isso, as rampas de aquecimento podem ser difíceis de reproduzir em diferentes equipamentos, o que pode implicar em uma completa nova otimização da metodologia. Outro complicador que deve ser levado em consideração é a idade do aparelho, fazendo com que a irradiação de micro- 
ondas produzida pelo magnetron seja prejudicada e com que experimentos em equipamentos similares ou no mesmo aparelho em épocas diferentes apresentem diferenças em rampas de temperatura.

Assim, de especial importância está a homogeneidade do campo elétrico na cavidade do reator. Enquanto em solução de baixa tangente de perda esta questão seja de menor relevância, a utilização de soluções com alta absortividade em micro-ondas pode conduzir a "hot spots”, pois nestes casos há uma menor homogeneidade do campo e em conseqüência da temperatura. Trabalhos demonstram que estes "hot spots" localizam-se próximos da barra magnética, em função de uma maior concentração do campo elétrico neste ponto onde a temperatura não pode ser medida.

No que se refere à escala da reação a ser reproduzida, deve ser salientado que experimentos em volumes de $1 \mathrm{~mL}$ e $3 \mathrm{~mL}$ apresentam rampas de aquecimento diferentes, onde aquela que apresenta maior volume aquece mais rapidamente. Esta observação além de contra-intuitiva vai contra o aprendizado diário com reações em aquecimento convencional, onde um aumento da escala conduz a taxas menores de aquecimento do meio reacional. A razão para este fenômeno está na maior capacidade de dissipação da energia irradiada na forma de calor. Por exemplo, a irradiação de $1 \mathrm{~mL}$ de acetonitrila a 30 $\mathrm{W}\left(25^{\circ} \mathrm{C}\right)$ é capaz de absorver apenas $0,39 \mathrm{~W}$, enquanto nas mesmas condições $3 \mathrm{~mL}$ são capazes de absorver 1,19 W e 1,93 W, por uma solução de $5 \mathrm{~mL}$.

Estas curvas de aquecimento, como foi demonstrado, são função de todas as variáveis intrínsecas de um experimento de micro-ondas, e podem ser completamente diferentes dependendo da potência, escala e solventes/ reagentes utilizados, podendo afetar profundamente os resultados. Outro fator importante é a avaliação do desempenho do magnetron. Para tal, é possível escolher uma reação padrão bem conhecida do laboratório ou até mesmo um solvente, de preferência de tangente de perda intermediária, e avaliar com o passar do tempo a curva de aquecimento deste sistema. Um aumento do tempo implica uma perda de desempenho do magnetron.

\section{Conclusão}

Com o exposto, esperamos ter deixado claro que o planejamento de um experimento de micro-ondas deve ser realizado com cuidado no que diz respeito à reprodutibilidade deste experimento, de modo que o máximo de informações deve ser relatado na parte experimental especialmente o tempo de decorrido para atingir a temperatura do experimento, e não apenas a temperatura final.

\section{Agradecimentos}

Agradecemos as agências de fomento FAPERJ, CNPq, FINEP e CAPES pelo apoio financeiro ao desenvolvimento de projetos no Laboratório de Biocatálise e Síntese Orgânica.

\section{Referências}

1. (a) Gedye, R.; Smith, F.; Westaway, K.; Ali, H.; Baldisera, L.; Laberge, L.; Rousell, J.; Tetrahedron Lett. 1986, 27, 279. (b) Giguere, R. J.; Bray, T. L.; Duncan, S. M.; Majetich, G.; Tetrahedron Lett. 1986, 27, 4945.

2. Gedye, R. N.; Wei, J. B.; Can. J. Chem. 1998, 76, 525.

3. Neas, E.; Collins, M.; Introduction to Microwave Sample Preparation: Theory and Practice, American Chemical Society, 1998.

4. (a) De La Hoz, A.; Diaz-Ortiz, A.; Moreno, A.; Chem. Soc. Rev. 2005, 34, 164. (b) Kuhnert, N. Angew. Chem. Int. Ed. 2002, 41, 1863. (c) Saillard, R.; Poux, M.; Berlan, J.; Audhuypeaudecerf, M.; Tetrahedron 1995, 51, 4033. (d) Herrero, M. A.; Kremsner, J. M.; Kappe, C. O.; J. Org. Chem. 2008, 73, 36.

5. (a) Chemat, F.; Esveld, E.; Chem. Eng. Technol. 2001, 24, 735. (b) Orrling, K.; Nilsson, P.; Gullberg, M.; Larhed, M.; Chem. Commun. 2004, 790. (c) Zhang, X.; Hayward, D. O.; Mingos, D. M. P.; Cal. Lett. 2003, 88, 33.

6. (a) Loupy, A.; Maurel, F.; Sabati-Gogov, A.; Tetrahedron 2004, 60, 1683. (b) De La Hoz, A.; Diaz-Ortiz, A.; Moreno, A.; Curr. Org. Chem. 2004, 8, 903. (c) Hayes, B. L.; Aldrichim. Acta 2004, 37, 66. (d) Razzaq, T.; Kremsner, J. M.; Kappe, C. O.; J. Org. Chem. 2008, 73, 6321. (e) Bougrin, K.; Bannani, A. K.; Tetouani, S. F.; Soufiaoui, M.; Tetrahedron Lett. 1994, 35, 8373.

\section{Marcella C. Flores', Carla C. Diederichs' ${ }^{1}$, Louise F Matos' ${ }^{1}$, Leandro S. M. Miranda' \& Rodrigo O. M. A. Souza ${ }^{1 *}$}

${ }^{1}$ Laboratório de Biocatálise e Síntese Orgânica, Instituto de Química, Universidade Federal de Rio de Janeiro, CT Bloco A, Rio de Janeiro, CEP 22941-909.

*e-mail: rodrigosouza@iq.ufrj.br 